\title{
Boas práticas de enfermagem voltadas à gravidez na adolescência: relato de experiência
}

\author{
Good nursing practices focused on teenage pregnancy: experience report
}

Buenas practicas de enfermería centradas em embarazo adolescente: informe de experiência

Nicole Râmilly de Oliveira Lameira ${ }^{1 *}$, Marina Pereira Queiroz dos Santos ${ }^{1}$, Leticia de Souza Rodrigues ${ }^{1}$, Tháyna Maressa Santos de Souza ${ }^{1}$, Juliana Conceição Dias Garcez ${ }^{1}$, Márcia Andrea da Gama Araújo ${ }^{1}$, Jorgeany Soares Parente ${ }^{1}$, Ianny Ferreira Raiol ${ }^{1}$, Rubenilson Caldas Valois ${ }^{1}$.

\section{RESUMO}

Objetivo: Realizar uma ação educativa com acadêmicos de enfermagem sobre boas práticas de enfermagem na gravidez de adolescentes. Relato de Experiência: Trata-se de um estudo qualitativo descritivo do tipo relato de experiência, ocorrido no Centro Universitário Metropolitano da Amazônia em Belém-PA em outubro de 2019. Participaram da ação vinte e oito acadêmicos do oitavo período do curso de graduação em enfermagem. A ação educativa ocorreu em etapas, inicialmente utilizou-se a Técnica de Livre Associação das Palavras (TALP); posteriormente ocorreu a explanação da temática; no terceiro momento ocorreu a discussão livre do tema abordado e por fim, repetiu-se a TALP com o intuito de comparar as palavras evocadas. Após a análise de similitude realizada com auxílio do IRAMUTEQ $\AA$, surgiram dois grafos com representação das coocorrências das palavras evocadas na TALP. Percebeu-se que os acadêmicos estavam bem instruídos sobre as atribuições, captações, acompanhamentos, tratamentos e prevenções de agravos acerca do prénatal, porém quando indagados sobre este atendimento direcionado a gravidez na adolescência as contraposições se tornaram divergentes. Considerações Finais: A ação educativa refletirá na formação do profissional de saúde a partir da reflexão da temática que resultará no auxílio adequado no manejo da gestação na adolescência.

Palavras-chave: Gravidez na adolescência, Adolescente, Cuidado pré-natal.

\begin{abstract}
Objective: To conduct an educational action with nursing students about good nursing practices in adolescent pregnancy. Experience Report: This is a qualitative descriptive study of the experience report type, which took place at the Metropolitan University Center of Amazonia in Belém - PA in October 2019. Twenty-eight students from the eighth period of the undergraduate nursing program participated in the action. The educational action took place in stages, initially the Free Word Association Technique (TALP) was used; later the explanation of the theme occurred; In the third moment there was a free discussion of the theme and finally, the TALP was repeated in order to compare the evoked words. After the similarity analysis performed with the aid of IRAMUTEQ $\AA$, two graphs with representation of the cooccurrences of the words evoked in the TALP appeared. It was noticed that the academics were well educated about the attributions, uptake, follow-up, treatment and prevention of prenatal disorders, but when asked about this care directed to teenage pregnancy, the opposing positions became divergent. Final Considerations: The educational action will reflect on the training of health professionals from the reflection of the theme that will result in adequate assistance in the management of pregnancy in adolescence.
\end{abstract}

Keywords: Teenage pregnancy, Teenager, Prenatal care.

${ }^{1}$ Centro Universitário Metropolitano da Amazônia (UNIFAMAZ), Belém- Pará.

*E-mail: nicolelameira@gmail.com

SUBMETIDO EM: 11/2019 


\section{RESUMEN}

Objetivo: realizar una acción educativa con estudiantes de enfermería sobre buenas prácticas de enfermería en el embarazo adolescente. Informe de Experiencia: Este es un estudio descriptivo cualitativo del tipo de informe de experiencia, que tuvo lugar en el Centro Universitario Metropolitano de Amazonia en Belém - PA en octubre de 2019. Veintiocho estudiantes del octavo período del programa de pregrado de enfermería participaron en la acción. La acción educativa se llevó a cabo en etapas, inicialmente se utilizó la técnica de asociación de palabras libres (TALP); luego ocurrió la explicación del tema; En el tercer momento hubo una discusión libre sobre el tema y finalmente, se repitió el TALP para comparar las palabras evocadas. Después del análisis de similitud realizado con la ayuda de IRAMUTEQ $\AA$, aparecieron dos gráficos con representación de las coincidencias de las palabras evocadas en el TALP. Se observó que los académicos estaban bien educados sobre las atribuciones, la captación, el seguimiento, el tratamiento y la prevención de los trastornos prenatales, pero cuando se les preguntó acerca de esta atención dirigida al embarazo adolescente, las posiciones opuestas se volvieron divergentes. Consideraciones Finales: La acción educativa se reflejará en la capacitación de profesionales de la salud a partir de la reflexión del tema que dará como resultado una asistencia adecuada en el manejo del embarazo en la adolescencia.

Palabras clave: Embarazo adolescente, Adolescente, Atención prenatal.

\section{INTRODUÇÃO}

A adolescência é a etapa da vida compreendida entre a infância e a fase adulta, marcada por um complexo e dinâmico processo de crescimento, desenvolvimento, amadurecimento e de intenso aprendizado de vida. A Organização Mundial da Saúde (OMS) considera a adolescência como a segunda década da vida e a juventude, a faixa dos 15 aos 24 anos (BEATRIZ BB e MARIA FM, 2012).

Segundo Maria $C$ et al. (2014) a gravidez na adolescência é um momento de incompreensão, em que o risco à saúde da mãe e da criança é real, considerando que nem sempre o corpo da adolescente está preparado fisiologicamente para o desenvolvimento da gestação. Da mesma forma, para Laís G, et al. (2016) a descoberta da gestação pode representar para a adolescente um momento de conflitos, ansiedades e angústias, na medida em que passa a conviver com dois eventos estressores: a adolescência e a gestação. Dentre as consequências da gravidez na adolescência, citam-se maiores incidências de síndrome hipertensiva da gravidez, anemia, diabetes gestacional, complicações no parto, determinando aumento de morte materna e infantil. No tocante aos problemas com o recém-nascido, a gravidez em adolescentes está associada a taxas mais elevadas de baixo peso ao nascer, parto pré-termo, doenças respiratórias e tocotraumatismo, além de maior periodicidade de complicações neonatais e mortalidade infantil (WALTER F, et al., 2015).

Sobretudo, a falta de lazer; a desestruturação familiar; a necessidade de expressar amor e confiança; a falta de informação sobre o uso adequado dos métodos contraceptivos; ausência de educação sexual e a falta de programas de planejamento familiar nos serviços públicos podem ser fatores predisponentes a gravidez na adolescência (CLÁUDIA CB, 2015).

Além disso, a falta de informações sobre os benefícios do pré-natal e a vergonha em buscar cuidados médicos, principalmente entre adolescentes mais pobres, está relacionada à maior ocorrência de parto prematuro e baixo peso ao nascimento e ao pré-natal inadequado, que implica em menor número de consultas, início mais tardio e baixa realização de exames complementares (FÁTIMA R, et al., 2013). Sendo assim, a gestante adolescente que recebe uma assistência pré-natal adequada tem impacto positivo sobre 0 resultado materno e perinatal, chegando eventualmente a anular possíveis desvantagens típicas da idade precoce. Portanto, fica evidente a importância do acompanhamento pré-natal integral durante a gravidez na adolescência (RITA FC, 2011).

Segundo Ellen CB (2011), os estudos atuais já comprovaram que o pré-natal consegue minimizar os riscos da gravidez na adolescência, mas infelizmente o acesso das adolescentes aos serviços de pré-natal continua 
insuficiente. É importante que os programas de pré-natal implantem estratégias destinadas à captação precoce e atendimento adequado e oportuno.

Assim, as características da enfermagem, em suas práticas de cuidado, possuem uma aproximação com os propósitos do acolhimento. Portanto, a formação dos profissionais de saúde deve ser mais ampla e contextualizada para que o enfermeiro seja preparado para desenvolver o cuidado de forma integral, contemplando os aspectos fisiológicos e patológicos (CLEUNIR F, et al., 2017). Visto que, as boas práticas estão diretamente dando suporte ao desenvolvimento do cuidado, no entanto para que seja eficaz é necessário que o profissional tenha ações humanizadas para gerar bons resultados.

Nesta perspectiva teve-se como proposta realizar uma ação educativa com acadêmicos de enfermagem sobre boas práticas de enfermagem na gravidez de adolescentes.

\section{RELATO DE EXPERIÊNCIA}

Trata-se de um estudo qualitativo descritivo do tipo relato de experiência, realizado por acadêmicos do curso de Enfermagem do sexto período, como Trabalho de Conclusão de Disciplina (TCD) após uma ação educativa, ocorrida no Centro Universitário Metropolitano da Amazônia em Belém- PA. O público alvo foram os acadêmicos do curso de Enfermagem do oitavo período dessa mesma instituição. A ação educativa ocorreu no dia 16 de outubro de 2019, com duração de uma hora e participação de vinte e oito acadêmicos.

Essa ação educativa foi rica em conhecimentos e experiências sobre a gravidez na adolescência, assim como as boas práticas de cuidados à saúde voltadas a essa temática. A elaboração dos formulários com base nas palavras evocadoras sobre esse conteúdo, proporcionou uma visão ampla e técnica, no qual, observouse $o$ interesse dos acadêmicos sobre como saber interagir e lidar com situações que irão ser vivenciadas quando estiverem exercendo a profissão.

Em relação aos aspectos éticos, devido ao presente estudo ser um relato de experiência não houve necessidade de submissão e avaliação do Comitê de Ética em Pesquisa (CEP), de acordo com a resolução 466/12 do Conselho Nacional de Saúde (CNS) e Resolução oo 510 de 2016. As autorias das fontes pesquisadas foram respeitadas, referenciando os autores citados no texto e nas referências bibliográficas conforme a lei vigente que rege os direitos autorais no país.

Na primeira etapa, foi disponibilizado um formulário impresso para cada acadêmico alvo da ação, no qual, deveriam colocar quatro palavras ou frases que lembrassem primeiramente, com relação a cada palavra evocadora que era exposta no quadro por meio de slide. O formulário foi composto com um espaço para cada palavra evocada, enumerada de forma consecutiva. As palavras evocadoras foram: Pré-Natal; Adolescente; Gravidez e Atuação da enfermagem. A segunda etapa, tratou-se de uma apresentação, através de slides, demonstrando a fase da adolescência, como o diagnóstico de gravidez é compreendido e a importância da atuação do enfermeiro no pré-natal de modo a reduzir os riscos relacionados a adolescente gestante.

No terceiro momento, houve uma discussão sobre o tema discorrido, no qual os acadêmicos puderam expressar suas experiências de modo a fortalecer as ideias repassadas sobre a temática. E por fim, a última etapa pôde trazer reflexões aos acadêmicos, com o formulário, sendo novamente aplicado, a fim de observarmos as alterações nas palavras evocadas e suas perspectivas com relação ao assunto abordado, caracterizando, assim, a TALP.

As palavras coletadas foram introduzidas em planilha do Excel e transportadas para o IRAMUTEQ $®$, utilizando a análise de matriz e representadas em uma análise de similitude representadas na seção posterior.

Sendo assim, as palavras coletadas e analisadas a partir da similitude realizada com auxílio do IRAMUTEQ $\AA$, proporcionou o surgimento de dois grafos com representação das coocorrências das palavras evocadas na técnica de associação das palavras.

Na Figura 1, evidencia- se o grafo inicial, elaborado com as palavras do formulário disponibilizado para os acadêmicos no início da ação educativa. Dessa forma, observou-se que ocorreram duas palavras, nos quais 
formaram núcleos centrais e se ramificam outras que apresentam expressões significativas. Na primeira palavra central "cuidado" ramificam- se as expressões "humanização", "atenção", "consulta", "pré-natal", "orientação", "acompanhamento", "responsabilidade", "segurança”, "acolhimento", "amor", "felicidade", "medo", "parto", "bebê" e "mãe". Em seguida, na palavra central "prevenção", subdivide- se as expressões "saúde", "recém-nascido", "sexo", "descobertas", "gravidez", "alegria" e "rebeldia". Abrangendo a essa percepção da análise do grafo textual, observou-se que as palavras cuidado e prevenção estão em foco central, podendo ser interpretadas como dependentes e complementares uma da outra, e as outras palavras que se ramificam integram-se agregando valor às centrais.

Figura 1 - Primeira análise de similitude utilizando a técnica TALP a partir do software IRAMUTEQ ®, 2019.

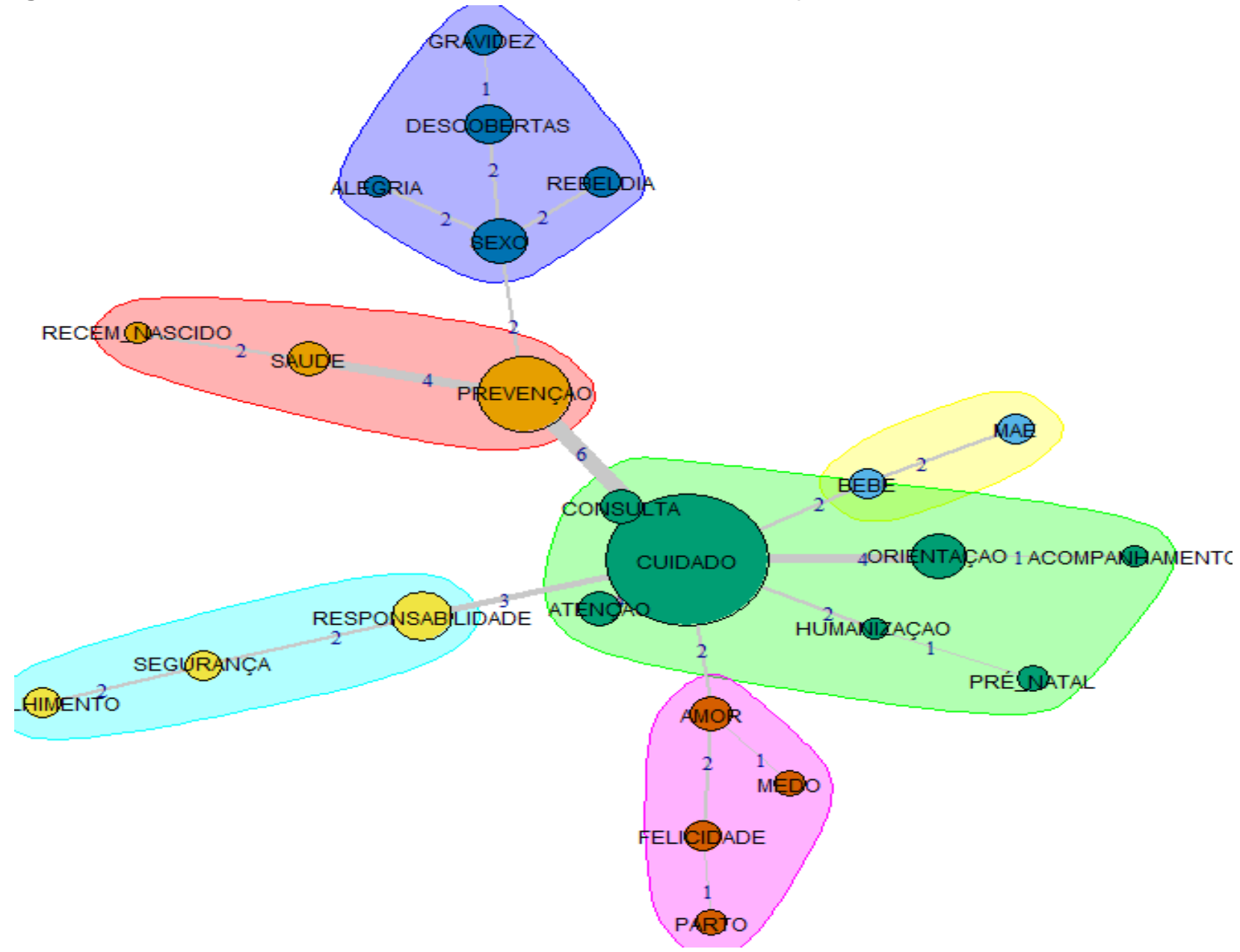

Fonte: LAMEIRA NRO, et al., 2019.

Na Figura 2, evidencia- se o grafo final, elaborado com as palavras do formulário disponibilizado para os acadêmicos no final da ação educativa.

Dessa forma, observou-se que ocorreram quatro palavras, nos quais formaram núcleos centrais e se ramificam outras que apresentam expressões significativas.

$\mathrm{Na}$ primeira palavra central "cuidado" subdivide- se as significantes "empatia", "parto", "pré-natal", "responsabilidade", "segurança", "prevenção", "educação", "assistência”, "importante", "fundamental", "consulta", "família", "acompanhamento", "conversar", "orientação", "humanização" e "ações". Posteriormente, o termo "atenção" ramifica- se com as expressões "amor", "diálogo", "respeito", "planejamento", "cuidar", "conflito", "mudanças", "frágil" e "hormônios". Em seguida, o termo central "ansiedade" ramifica- se em "aprendizagem", "aprendiz", "descobertas", "sexo", "sexualidade", "medo" e "aborto espontâneo". Por fim, a palavra "acolhimento" se expressa em "fragilidade", "conhecimento", "saúde", "necessário" e "vida". 
Figura 2- Segunda análise de similitude utilizando a técnica TALP a partir do software IRAMUTEQ $\circledast, 2019$.

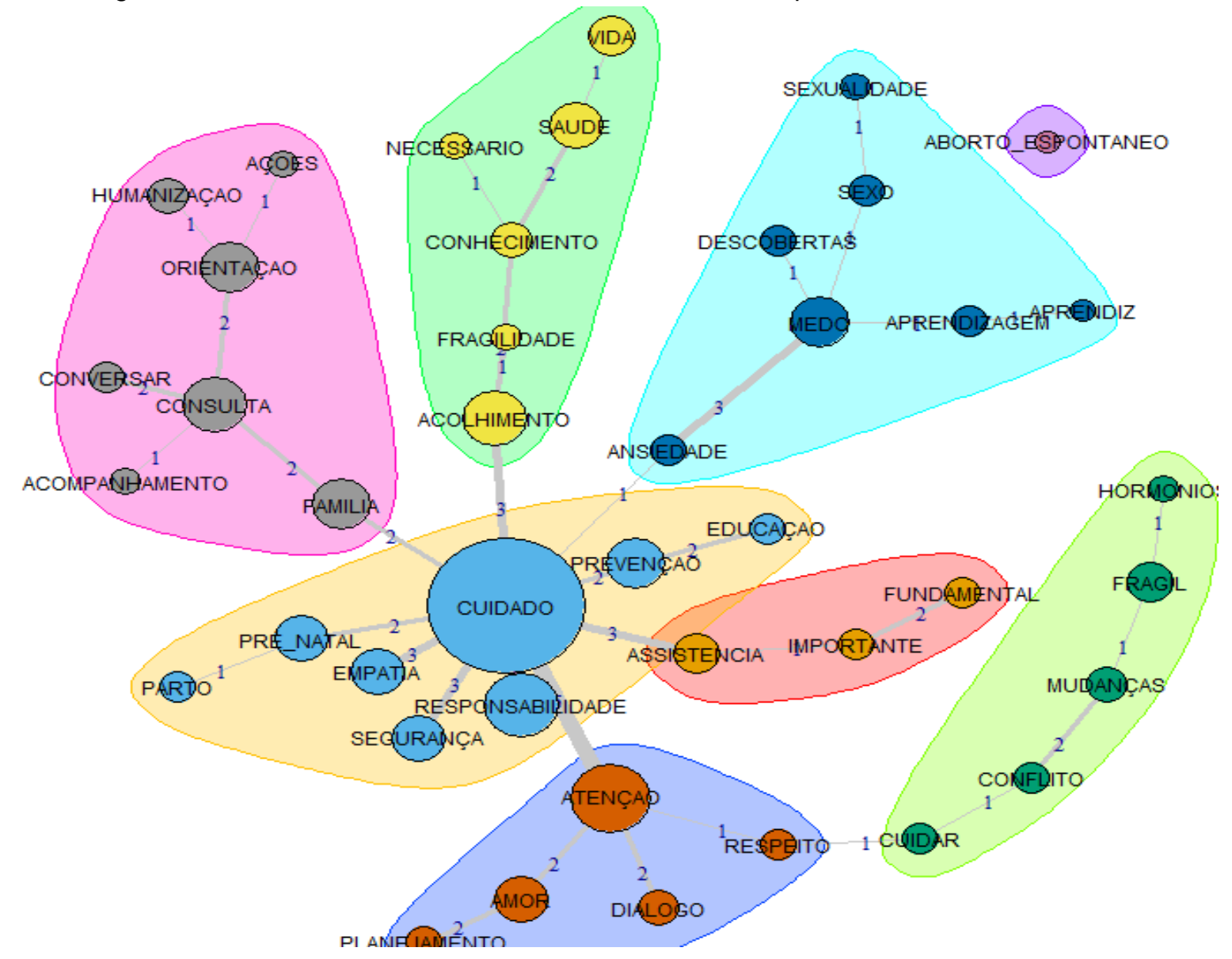

Fonte: LAMEIRA NRO, et al., 2019.

Percebeu-se que os acadêmicos estavam bem instruídos sobre as atribuições, captações, acompanhamentos, tratamentos e prevenções de agravos acerca do pré-natal, porém quando indagados sobre este atendimento direcionado a gravidez na adolescência as contraposições se tornaram divergentes, sendo relacionadas a questão psicológica, econômica, familiar e cultural. No entanto, as considerações se complementavam de modo a enriquecer os conhecimentos.

\section{DISCUSSÃO}

A atividade foi realizada em quatro etapas a partir da Técnica de Associação Livre das Palavras (TALP). Esta, é um instrumento que se estrutura através da evocação de palavras previamente definidos, levando em consideração a amostra ou função do objeto em pesquisa (COUTINHO MP, 2017).

Posterior a aplicação da técnica, as evocações foram avaliadas pelo software IRAMUTEQ ® (Interface de $R$ pour les Analyses Multidimensionnelles de Textes et de Questionnaires), para verificação de assimilação do conteúdo proposto através da análise de similitude. Esta, fundamenta-se na teoria dos grafos e possibilita distinguir o seu resultado através da correlação entre as palavras, dentro de um corpus textual, diferenciando as partes comuns e as específicas (SOUZA M, et al., 2018).

Destarte, de acordo com os dois grafos gerados foi possível identificar a prevalência da palavra "cuidado" como núcleo principal à realização das boas práticas do enfermeiro na atenção da gravidez na adolescência, com base na integralidade, ultrapassando as dimensões das técnicas a partir de espaços estimuladores do diálogo, que valorizem a história de vida, a crença e a cultura de cada indivíduo, proporcionando o desenvolvimento do cuidado (SONIA A, et al., 2014). 
Em vista disso, a ocorrência da gravidez na adolescência não é recente, no entanto, emergem novas formas de compreendê-la e aceitá-la segundo o pensamento da sociedade moderna. Atualmente, é compreendida como um processo que interrompe o crescimento e o amadurecimento da adolescente, resultando em perdas de oportunidades (MILLA W, et al., 2015).

Por conseguinte, as condições socioeconômicas e a realidade social na qual essas adolescentes estão inseridas, determinam de que forma estas irão atravessar esse momento. Assim, é necessário um olhar mais crítico e atencioso dos profissionais que irão atuar com essas adolescentes gestantes para os motivos que levaram essas adolescentes a serem mães tão prematuramente como também, observar de que forma esses sujeitos estão passando pela fase da adolescência, social e economicamente (DAYANE NC, 2017).

\section{CONSIDERAÇÕES FINAIS}

É perceptível que são muitas as consequências da gravidez na adolescência e elas podem afetar aspectos sociais, psicológicos e biológicos da vida e do bem-estar das mulheres jovens, influenciando um processo de desconstrução nas relações interpessoais e sua inserção no mercado de trabalho. Logo, o papel da enfermagem é acolher com escuta qualificada e responsabilização diante das especificidades das demandas, valorizando o contexto em que estas são geradas. Portanto, há de se reconhecer a importância e necessidade do processo vivenciado pelo acadêmico, uma vez que o torna preparado para enfrentar a realidade, pois, é no pré-natal que as adolescentes grávidas recebem as informações e orientações sobre determinados cuidados necessários para uma gravidez saudável, e que sem dúvida a assistência quando adequada, é um dos fatores mais benéficos para uma gestação bem-sucedida.

\section{REFERÊNCIAS}

1. BEATRIZ BB, MARIA FM. A adolescente grávida na percepção de médicos e enfermeiros da atenção básica. Escola Anna Nery, 2012; 16.

2. CLÁUDIA CB. Gravidez na adolescência e (in)sucesso escolar: Um estudo qualitativo em escolas secundárias de Benguela. Dissertação (Mestrado em Administração e Gestão da Educação) - Departamento de Psicologia e Educação, 2015.

3. CLEUNIR $F$, et al. Fatores que possibilitam a atuação do enfermeiro na atenção pré-natal. Res.: fundam. Care, 2017; 9(4): 978-983.

4. COUTINHO MP. A técnica de associação livre de palavras sobre o prisma do software tri-deux-mots. Revista Campo do Saber, 2017; 3(1).

5. DAYANE NC. Gravidez na adolescência: Caminhos entre projetos de vida e a realidade. Trabalho de Conclusão de Curso. Universidade Federal do Rio Grande do Norte, Natal, 2017.

6. ELLEN CB. Assistência de enfermagem a adolescentes grávidas. Monografia apresentada para a conclusão do Curso de Graduação de Enfermagem pela Universidade Salgado de Oliveira, 2011.

7. FÁTIMA R, et al. Pré-natal na adolescência: uma revisão crítica. Adolesc. Saúde, 2013; 10(1): 43-50.

8. LAí́S G, et al. Assistência de enfermagem às adolescentes gestantes sob a Ótica de Callista Roy. Enferm, 2016; 7(3/4): 46-50.

9. MARIA C, et al. Assistência pré-natal à adolescente e os atributos da Atenção Primária à Saúde. Rev. Latino-Am. Enfermagem, 2014; 22(1).

10. MILLA W, et al. A prevenção da gravidez na adolescência na visão de adolescentes. Texto Contexto Enferm, Florianópolis, 2015; 24(1): 30-7.

11. RITA FC. Atenção ao pré-natal na adolescência. Dissertação (Mestre em Ciências) - Práticas Sociais em Enfermagem e Saúde. Faculdade de Enfermagem da Universidade Federal de Pelotas, 2011.

12. SONIA A, et al. Práticas de cuidado: o papel do enfermeiro na atenção básica. Rev. Enferm, 2014.

13. SOUZA M, et al. O uso do software IRAMUTEQ na análise de dados em pesquisas qualitativas. Rev. Esc. Enferm, 2018.

14. WALTER F, et al. Complicações da gravidez na adolescência: revisão sistemática da literatura. Einstein, 2015. 